\title{
Improving Fluorometric Determination of Water Content in Aprotic Solvents
}

\author{
Katarzyna Kłucińska ${ }^{1}$ - Patryk Rzepiński ${ }^{1}$ - Maciej Mazur ${ }^{1}$ - Michal K. Cyrański ${ }^{1}$ • \\ Krzysztof Maksymiuk $^{1} \cdot$ Agata Michalska $^{1}$ (D)
}

Received: 31 May 2017 / Accepted: 4 August 2017 /Published online: 24 August 2017

(C) The Author(s) 2017. This article is an open access publication

\begin{abstract}
A fluorometric method allowing quantification of minute amounts of water in highly lipophilic solvents is proposed. This approach takes advantage of resonance energy transfer between 4-methylumbelliferone and Sudan I. The conditions required to observe effective energy transfer have been elucidated. The emission band of the donor molecule (4methylumbelliferone) depends strongly on the water content - its position is affected by protolytic reactions occurring between the fluorescent dye and water in lipophilic solvent media. In consequence, the extent of overlap between the emission band of the donor and absorption peak of the acceptor scales with the water concentration. The sensitivity of this optical system can be considerably increased by addition of organic bases. The dependence of the emission band of 4methylumbelliferone/Sudan I system on minute amount of water present in lipophilic solutions opens up possibility of determination of water content in these media at the unique wavelength, in a turn on mode. The presented system was successfully applied to determine water in model commercial oil samples. It was shown that using herein proposed method the increase of water contents in the sample can be observed as increase of the emission intensity, despite the native fluorescence of the vegetable oil samples. For these samples, a good correlation with golden standard Karl Fischer titration was obtained.
\end{abstract}

Electronic supplementary material The online version of this article (doi:10.1007/s12161-017-1019-7) contains supplementary material, which is available to authorized users.

Agata Michalska

agatam@chem.uw.edu.pl

1 Department of Chemistry, Warsaw University, Pasteura 1, 02-093 Warsaw, Poland
Keywords Water determination $\cdot$ RET . 4-Methylumbelliferone · Sudan I · Aliphatic solvents · Fluorometry $\cdot$ Vegetable oils

\section{Introduction}

Quantification of water in organic solvents is important for many applications, including industrial ones. The standard method for water determination is coulometric Karl Fischer titration (Dantan et al. 2000). The electrochemical approach is generally highly reliable; however, in some cases, especially for relatively small water concentrations and highly lipophilic, aprotic solvents, it may potentially be sensitive to, e.g., sample acquisition due to tendency of water to spontaneously accumulate close to more hydrophilic surfaces - e.g., glassware. This effect may result in inaccurate determination results (Dantan et al. 2000; Gergen et al. 2006). Moreover, the Karl Fischer titration method is difficult to be implemented as a part of, e.g., industrial or technological process, or to be used to continuously monitor any occurrence of water in sample media. Therefore, different alternative approaches for quantification of water in organic solvents have been proposed, including advanced techniques like NMR (Sun et al. 2008), infrared spectrometry (Li and Paecy 1997; Garrigues et al. 1993), and UV/vis spectrometry (Langhals 1990; Pinheiro et al. 2006). The fluorometric approach is also attractive for water quantification in organic solvents (e.g., Yunxiang and Xin 1984; Ding et al. 2013; Gao et al. 2009; Liu et al. 1999; Kłucińska et al. 2015), provided that the sensitive probe of emission properties dependent on the amount of water present in the sample is available. Different dyes have been proposed/tested in this respect, most of them were tailor made (synthesized) for water determination purpose, and in most cases, the increase of water contents in the sample was accompanied with 
decrease (quenching) of emission signal (e.g., Yunxiang and Xin 1984; Gao et al. 2009; Niu et al. 2010), which is clearly unfavorable for practical purposes.

In our recent work, we have demonstrated possibility of application of relatively popular, commercially available fluorophore 4-methylumbelliferone (4-MU) as sensitive, turn-on, water probe (Kłucińska et al. 2015). The sensing mechanism proposed was taking advantage of dependence of the emission spectrum of 4-MU on acid/base reactions (e.g., Nakashima et al. 1972; Bardez et al. 1992). The approach proposed (Kłucińska et al. 2015) was based on a protolytic reaction between 4-MU and water (acting as a base) occurring in a lipophilic solvent, leading to formation of highly fluorescent deprotonated 4-MU characterized with emission at about $450 \mathrm{~nm}$. This approach was shown to be highly effective, especially in minute water concentrations, also in the presence of other lipophilic acids or bases in the system, offering higher sensitivity, compared to classical Karl Fischer approach (Kłucińska et al. 2015).

The emission at about $450 \mathrm{~nm}$ is characteristic for different substances including mineral or vegetable oils, which can be a problem for 4-MU-based quantification method (Kłucińska et al. 2015). Thus, from practical applications' point of view, it would add value to the method, if the correlation between water contents and emission signal can be also observed at other wavelengths, without the adverse effect on analytical performance of the method.

Therefore, the aim of this work was to further improve the proposed system, preserving the principle of the determination related to protolytic 4-MU reaction, however, to offer possibility of shifting the emission to other wavelengths. To achieve this goal, we propose to apply a transducer dye of spectrum independent of the protolytic reactions but able to interact with 4-MU to yield formation of a new, water contentdependent, emission peak. The herein proposed novel approach based on application of a mixture of two different dyes, one applied as reporter and the other one as optical transducer, to our best knowledge has not been applied for water determination in lipophilic solvents.

\section{Experimental}

\section{Reagents}

Hexadecane (HD), 4-methylumbelliferone (4-MU), Sudan I, HYDRANAL $\AA$ - Composite $5 \mathrm{~K}$ (Titration), HYDRANAL ${ }^{\circledR}$ - Medium K, HYDRANAL $®$ - Water Standard 10.0, octanoic acid, and molecular sieves were from Aldrich (Germany). Octylamine, hydrochloric acid, and sodium hydroxide were of analytical grade and were obtained from POCh (Gliwice, Poland). Samples of vegetable-sunflower oil and olive oil, were purchased in a local store.
Doubly distilled and freshly deionized water (resistance 18.2 $\mathrm{M} \Omega \mathrm{cm}$, Milli-Qplus, Millipore, Austria) was used throughout this work.

All solvents were dried over freshly activated molecular sieves (MS 4A).

\section{Apparatus}

Fluorometric experiments were performed using a spectrofluorimeter Cary Eclipse (Varian). After exposure at an excitation wavelength, unless otherwise stated, equal to $330 \mathrm{~nm}$, emission intensity was recorded within the range from $350 \mathrm{~nm}$ (or $370 \mathrm{~nm}$ ) to $600 \mathrm{~nm}$. The slits used were $5 \mathrm{~nm}$ both for excitation and emission, while the detector voltage was maintained at $800 \mathrm{~V}$.

Biamperometric Karl Fischer (KF) titration in volumetric mode was performed using titration equipment: 716 DMS Titrino (Metrohm) and 728 Stirrer (Metrohm), and platinum electrodes $(400 \mathrm{mV})$ were used. Chloroform was added to the working solution as a solubility promoter so that the KF titration was conducted in the homogeneous solution. This procedure was found to give more reproducible results. The requirements of complete dissolution in KF method were also discussed earlier (Margolis 1995).

Emulsions were prepared using homogenizer Hielscher, model UP 200S.

\section{Powder X-ray Diffraction}

Hexane solutions of $1: 1.4 \mathrm{M}$ ratio Sudan I to 4methylumbelliferone mixture with or without presence of $\mathrm{NaOH}$ were prepared. After evaporation, the powder samples were analyzed with use of the X-ray powder diffraction method. The experiments were performed at room temperature on a Bruker D8 Discover diffractometer equipped with capillary stage, sealed tube with $\mathrm{Cu}$ anode, Goebel focusing mirror, and LynxEye detector. The initial $2 \theta$ range for the data collection was $5^{\circ}-50^{\circ}$. Further processing of the data was performed using DIFFRACEVA. To identify experimentally the obtained diffractograms, powder X-ray diffraction (PXRD) diffractograms of individual components of the mixtures were generated with use of CCDC Mercury software (Macrae et al. 2008) based on their known single-crystal structures. Since Sudan I forms three polymorphs, individual PXRD patterns of Sudan I and of 4-methylumbelliferone, both crystallized from hexane, were also recorded (see Fig. S2 and S3 in the ESI). The phase analysis revealed that Sudan I consists of $82 \%$ of the polymorph I (Oliveri et al. 1989), 8\% of the polymorph II (Liu et al. 1997), and 10\% of the polymorph III (Ferreira et al. 2013), whereas 4-methylumbelliferone exists in the anhydrous form (Panini et al. 2014). 


\section{Preparation of 4-MU or Sudan I Stock Solutions}

Dye stock solutions in tested solvents were prepared by dissolving $2 \mathrm{mg}$ of 4-MU or Sudan I in $80 \mathrm{ml}$ of hexadecane under sonication (cycle 0.5 , power $70 \%$ ) yielding a concentration $0.025 \mathrm{mg} / \mathrm{ml}$. Sonication was continued for another $5 \mathrm{~min}$. Then the mixture was left for $24 \mathrm{~h}$ to complete dissolution of the fluorophore. Thus, prepared stock solutions were used for fluorimetric measurements.

\section{Preparation of 4-MU, Transducer Dye, or Mixture Solutions}

Corresponding amounts of fluorophore (4-MU) and Sudan I were dissolved in hexadecane, under sonication (cycle 0.5 , power $70 \%$ ), to obtain molar ratio 4 -MU to Sudan $I=1.4: 1$. Concentration values were $4.5 \times 10^{-4} \mathrm{M}$ for the fluorophore and $3.2 \times 10^{-4} \mathrm{M}$ for Sudan I. In case of vegetable oil samples, dyes were dissolved in oil sample following the procedure described above.

\section{Optical Measurements}

Water was introduced to hexadecane containing reporter dye (4-MU) and optical transducer-Sudan I. To assure that introduced water is mixed with the dry solvent, the sample was sonicated for $60 \mathrm{~s}$. In case of commercial oil samples, after water introduction, and emulsion preparation, the samples were diluted with HD in ratio 1:2 (oil:HD).

For measurements, volumes of the sample were 2 or $3 \mathrm{ml}$ for $\mathrm{UV} / \mathrm{vis}$ or fluorescence measurements, respectively.

\section{Results and Discussion}

To explore the possibility of observing emission maximum related to protolytic reaction between 4-MU and water in lipophilic solvents at other wavelengths than characteristic for 4-MU emission (maximum observed at $450 \mathrm{~nm}$ ), interactions between different optically active species in the sample need to be taken into consideration. The approach proposed in this work is based on application of a reporter dye (4-MU) of proven sensitivity in "turn on" mode for increasing water contents in lipophilic solvents and the optical transducer (Sudan I). The important requirement for an optical transducer is that this compound is characterized with absorption spectrum independent of $\mathrm{pH}$, good solubility in lipophilic environment, and presence of fluorescence signal. The key requirement is that the absorption band of the optical transducer overlaps with the emission band of 4-MU in water containing lipophilic solvents. Provided that these conditions are fulfilled, the energy transfer between the reporter and transducer can be observed resulting in formation of a signal intensity dependent on water content in the lipophilic solvent. It seems that the excellent candidate to be transducer dye for 4-MU reporter can be Sudan I-dye soluble in lipophilic media.

Figure 1a shows emission spectra recorded for 4-MU in different conditions. As reported before (Kłucińska et al. 2015), the 4-MU in neat hexadecane reveals very weak emission band with a maximum at $375 \mathrm{~nm}$. After addition of a base (alkyl amine) to hexadecane, the emission band of 4-MU shifts to $450 \mathrm{~nm}$ and considerably increases its intensity. This occurs due to deprotonation of 4-MU (similarly as it is observed in water solutions of 4-MU containing $\mathrm{NaOH}$ ). On the other hand, addition of a carboxylic acid to hexadecane (e.g., octanoic acid) results in increase of intensity and shift of the 4-MU band to $485 \mathrm{~nm}$. Here, this spectral shift is due to protonation of 4-MU molecules. These results confirm that while neutral 4-MU molecule is rather weakly fluorescent, its protonation or deprotonation results in red shift of emission band and changes in its intensity. Similarly, addition of minute amount of water to hexadecane phase affects the spectrum of 4-MU. As seen at Fig. 1a, the spectrum reveals emission with a maximum at $450 \mathrm{~nm}$ and significant intensity. This behavior is explained assuming that water acts as a base transforming 4MU into deprotonated form (Kłucińska et al. 2015).

With this information in hand, one can consider the properties of the candidate for a transducer dye molecule, Sudan I. The absorption spectrum of Sudan I dissolved in hexadecane is shown in Fig. 1b. As it can be seen, the spectrum is characterized with presence of a broad absorption band ranging from 420 to $520 \mathrm{~nm}$, with three maxima, at about 420, 475, and $500 \mathrm{~nm}$. The dye reveals a relatively weak emission with maximum at $550 \mathrm{~nm}$ after excitation at $470 \mathrm{~nm}$ (Fig. 1b) (Muthu Prabhu et al. 2010). Within the range of experimental error, essentially the same absorption and emission spectra have been recorded also in hexadecane spiked with base (amine) or acid, confirming that Sudan I spectral properties are independent of change of protolytic properties of its microenvironment (Fig. 1b).

Next, we have studied the emission characteristics of both dyes together (4-MU and Sudan I) in lipophilic media. When the chromophores are both present at 1.4:1 $\mathrm{M}$ ratio Sudan I to 4-MU in neat hexadecane and the system is excited at $330 \mathrm{~nm}$ (excitation wavelength of 4-MU), one can observe practically no emission signal (Fig. 2). This is not surprising since the fluorescence of 4-MU in neat hexadecane is very low (Kłucińska et al. 2015). With increase of octylamine contents - acting as protolytic agent on 4-MU (Kłucińska et al. 2015) in the system - the increase of emission bands at 450 and $540 \mathrm{~nm}$ is observed (Fig. 2). For concentration of amine $10^{-5}$ or $10^{-4} \mathrm{M}$, some increase of emission at about $450 \mathrm{~nm}$ is observed, i.e., at wavelength typical for deprotonated 4-MU. In the excitation spectra, increase of signal after addition of octylamine was also observed (Fig. S1). It should be stressed that similar dependencies were recorded also in the presence 
Fig. 1 a Emission spectra of 4MU recorded under different conditions: (black line) in dry hexadecane, (red line) in hexadecane containing $10^{-2} \mathrm{M}$ octanoic acid, (blue line) in hexadecane containing $10^{-3} \mathrm{M}$ octylamine, (green line) in hexadecane containing $10 \mu \mathrm{l}$ of water, (pink line) hexadecane containing $10^{-3} \mathrm{M} \mathrm{NaOH}$.

$\mathrm{Ex}=320 \mathrm{~nm}$. b Absorption and emission spectra of Sudan I in (black line) hexadecane, (red line) hexadecane containing $10^{-2} \mathrm{M}$ octanoic acid, and (blue line) hexadecane containing $10^{-2} \mathrm{M}$ octylamine. $\mathrm{Ex}=470 \mathrm{~nm}$ a

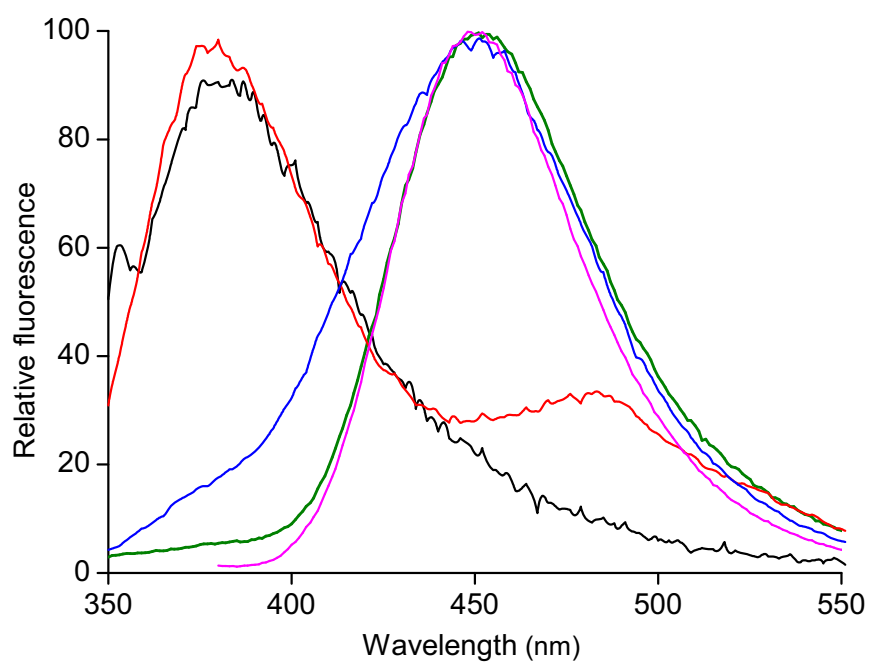

b

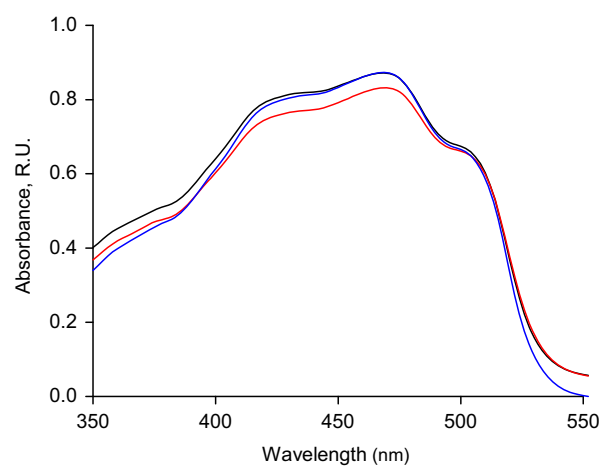

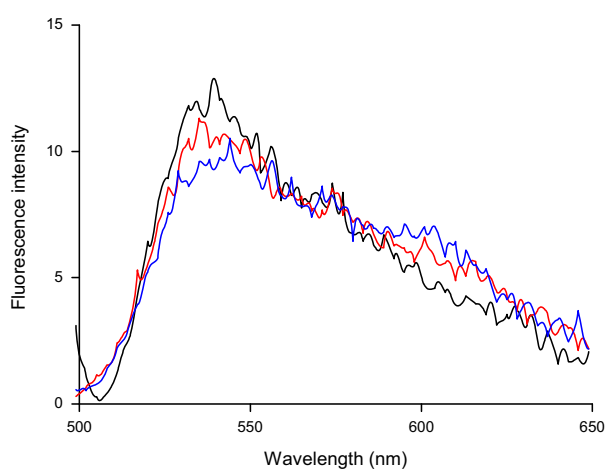

of other lipophilic amines: isopentylamine, octadecyl amine (results not shown).

The band at $450 \mathrm{~nm}$ is due to 4-MU emission, while the signal at $540 \mathrm{~nm}$ is attributable to Sudan I. It seems that the band at $540 \mathrm{~nm}$ results from resonance energy transfer from 4MU chromophore to Sudan I dye. This suggests that the chromophores must be in short distance in the lipophilic environment which is the sine qua non condition for the dipole-dipole interaction and energy transfer. We were interested therefore whether these two fluorophores, being relatively polar, may form conjugates in a lipophilic medium (and this is the reason for effective energy transfer) or it is the presence of water phase (e.g., in the form of emulsion droplets) that allows their close proximity. To answer these questions, the X-ray diffraction measurements have been performed.

\section{Solid-State Investigations}

To investigate molecular interactions of Sudan I and 4-MU in lipophilic media, we prepared their mixtures in the presence or in the absence of $\mathrm{NaOH}$ (added as aqueous solution, $50 \mu \mathrm{l}$ for $5 \mathrm{ml}$ of fluorophore solution in a hexadecane). After the solvent evaporation, the samples were investigated with use of the powder X-ray diffraction technique. The resulted diffractograms are presented in Figs. 3 and 4. To interpret them, we have simulated the patterns of individual

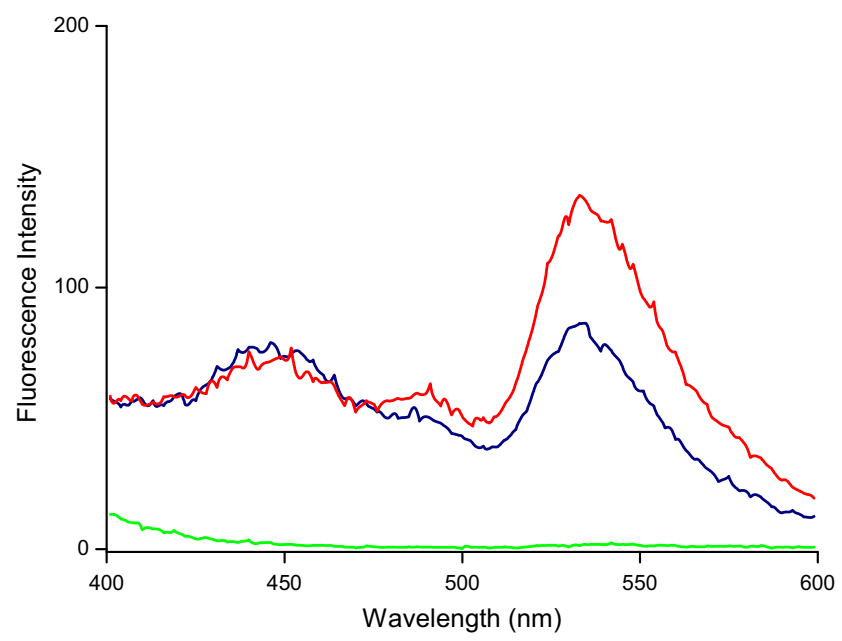

Fig. 2 Emission spectra of 4-MU and Sudan I in hexadecane, in the absence (green line) and in the presence of octylamine: (blue line) $10^{-5} \mathrm{M}$ octylamine, (red line) $10^{-4} \mathrm{M}$ octylamine 
Fig. 3 PXRD pattern for mixture of Sudan I and 4-

methylumbelliferone in the presence of $\mathrm{NaOH}$ (in black) and the simulated PXRD patterns for polymorph I of Sudan I (red), trona (blue), and MU monohydrate (green)

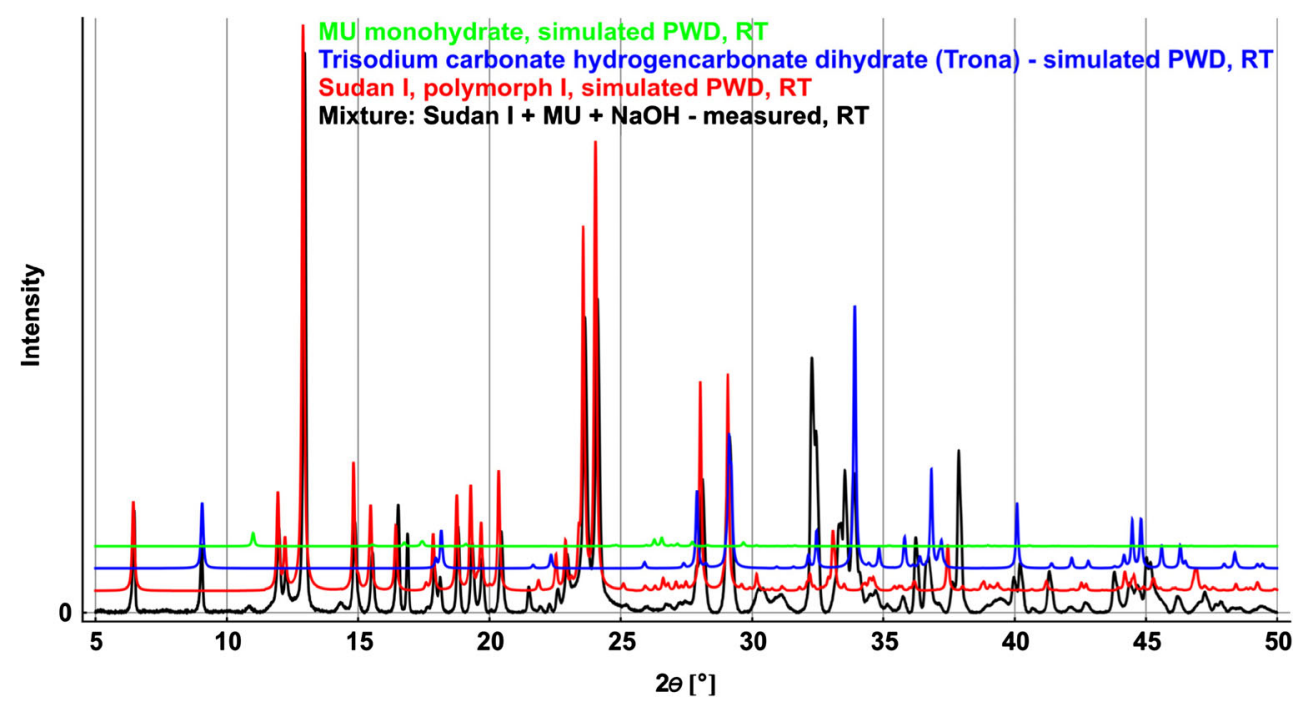

components of the mixture based on their single-crystal structures. The phase analysis of the mixture Sudan I and 4methylumbelliferone in the presence of $\mathrm{NaOH}$ revealed the coexistence of crystalline forms of Sudan I, polymorph I (Oliveri et al. 1989), the 4-MU hydrate (Panini et al. 2014), and $\mathrm{Na}_{2} \mathrm{CO}_{3} \cdot \mathrm{NaHCO}_{3} \cdot 2 \mathrm{H}_{2} \mathrm{O}$ (Candlin 1956) (see Fig. 3), whereas the base-free crystallization indicates the coexistence of crystalline forms of Sudan I, polymorph I (Oliveri et al. 1989), Sudan I, polymorph II (Liu et al. 1997), and 4methylumbelliferone (Panini et al. 2014). The simulated bands for Sudan I polymorph II are somewhat shifted due to temperature effect (Liu et al. 1997). Unfortunately, none of these patterns could be attributed to a phase where direct interactions of 4-MU and Sudan I at molecular scale are observed. Clearly, 4-MU and Sudan I do not form conjugates in the ground state; however, the excited state interactions cannot be excluded. It is worth noting that the hydration of 4-MU leads to strengthening of $\pi \ldots \pi$ stacking interactions between coumarin fragments in the solid state (Panini et al. 2014). In the case of the hydrated form, the $C^{\pi} \ldots C^{\pi}$ contacts yield approximately at $3.4 \AA$. This indicates that the molecules in the solution may effectively approach each other, what enables energy transfer processes, e.g., between 4-MU and Sudan I molecules.

\section{Water Determination Using 4-MU/Sudan I System in the Model Lipophilic Solvent}

In line with the results presented in our previous work (Kłucińska et al. 2015), the effect of introduction of water to
Fig. 4 PXRD pattern for mixture of Sudan I and 4-

methylumbelliferone (in black) and simulated PXRD patterns for Sudan I polymorph I (green), polymorph II (blue), and 4methylumbelliferone (red)

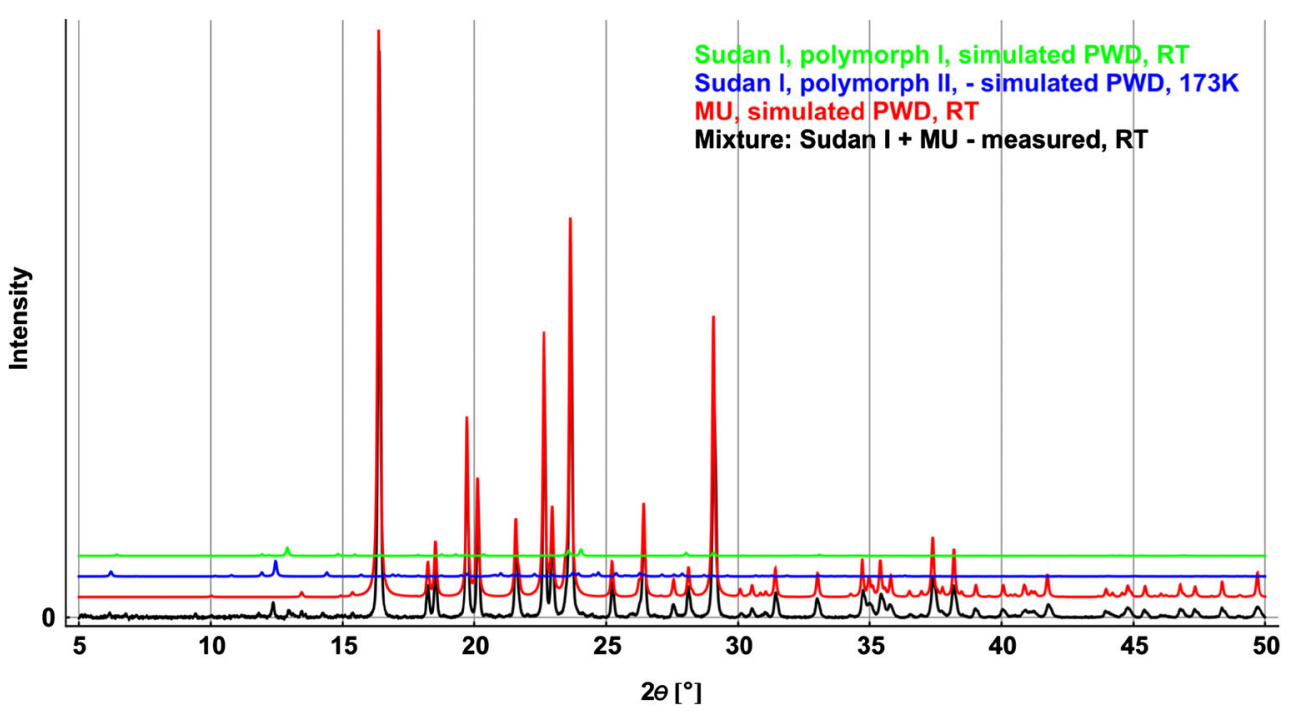


the model lipophilic solvent, hexadecane was studied. Water in this system is acting as a base, as discussed previously (Kłucińska et al. 2015). Thus, for herein proposed system of 4-MU receptor dye and Sudan I transducer dye, it is expected that increase of water contents in the sample will lead to increased overlap of emission spectra of 4-MU and absorption spectra of Sudan I, due to shift and enhancement of the reporter dye emission band.

As it can be seen in Fig. 5a, the increase of water content in hexadecane containing 4-MU and Sudan I results in increased emission intensity observed at $540 \mathrm{~nm}$, i.e., increase of the emission band characteristic for resonance energy transfer between 4-MU (in the presence of water) and Sudan I. For a higher concentration of water, also a peak at about $450 \mathrm{~nm}$ is formed. This peak is characteristic for deprotonated form of 4MU fluorophore (Fig. 5a).

The comparison of dependencies obtained by plotting emission intensity vs. water contents in the sample (within the range from 0 to $3.33 \% V / V$ ) is shown in Fig. 5 b. The emission peaks were observed at 530 and $450 \mathrm{~nm}$, with increase of emission corresponding to increase of water contents in the sample. Linear dependence of emission intensity on water contents was observed in the whole investigated range for both wavelengths stated above $\left(R^{2} @ 530=0.996\right.$ and $\left.R^{2} @ 450=0.990\right)$.

As it can be seen in Fig. $5 \mathrm{~b}$, the dependence read at $540 \mathrm{~nm}$ offers higher sensitivity of determination, i.e., higher change of emission for the same change of water contents, compared to the intensity read at $450 \mathrm{~nm}$. It should be stressed that the sensitivity of dependence for lower contents of water in the sample was similar for both wavelengths. It should be also added that the dependences plotted using emission intensity read at $450 \mathrm{~nm}$, shown in Fig. 5b, are equivalent to those reported by us earlier for the system containing 4-MU as the only dye in the lipophilic solvent (Kłucińska et al. 2015).

Thus, it can be concluded that the herein proposed approach based on application of combination of two dyes present in the sample offers a significant improvement of the highly sensitive method of fluorimetric determination of water contents in the lipophilic solvent samples. The high sensitivity offered by the system based on application of 4-MU (Kłucińska et al. 2015) was extended for higher contents of water in the samples, i.e., for the range inaccessible for the system using only one dye. It should be stressed that linear response range and detection limit were well comparable to the literature reports using other fluorimetric approaches for determination of water in organic solvents, e.g., in dioxane where linear response of the probe covered the water content to $4.00 \% V / V(\mathrm{DL}(\%)=0.019)$ ( Niu et al. 2010) or with the use of fluorescent nanospheres (Gao et al. 2009) (linear dependence of emission
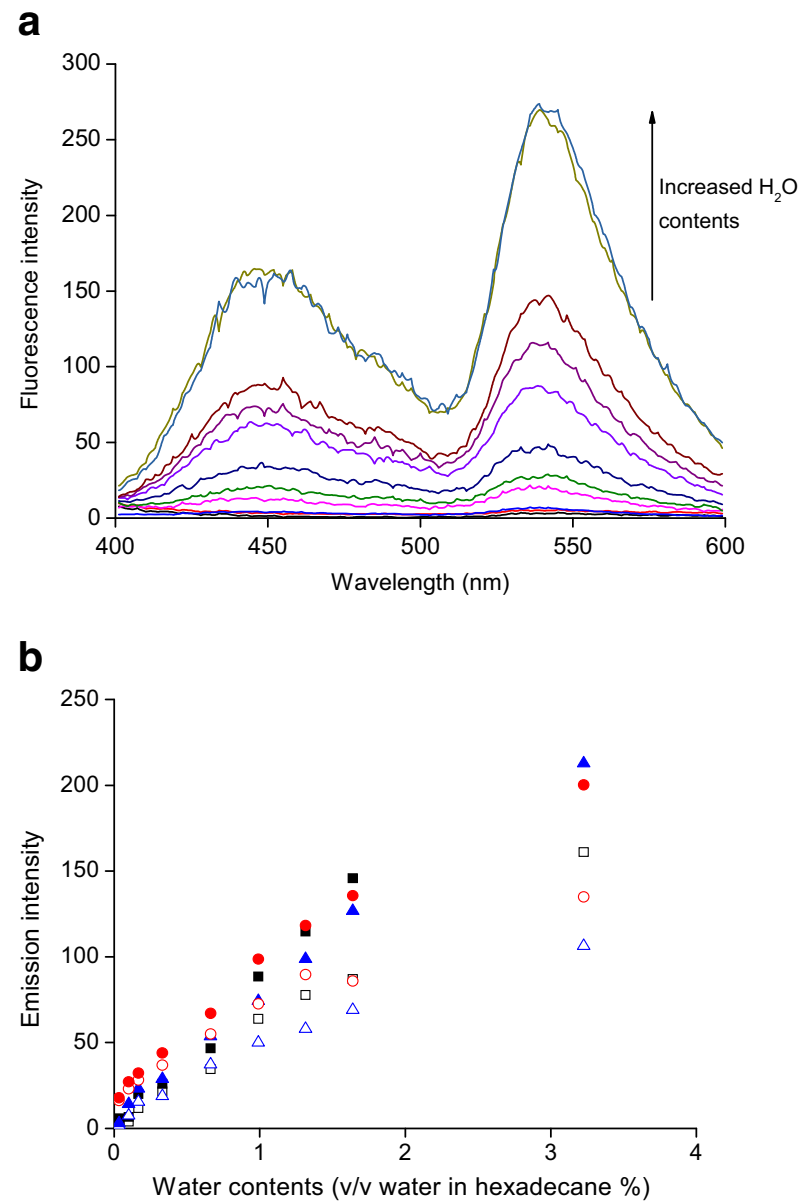

C

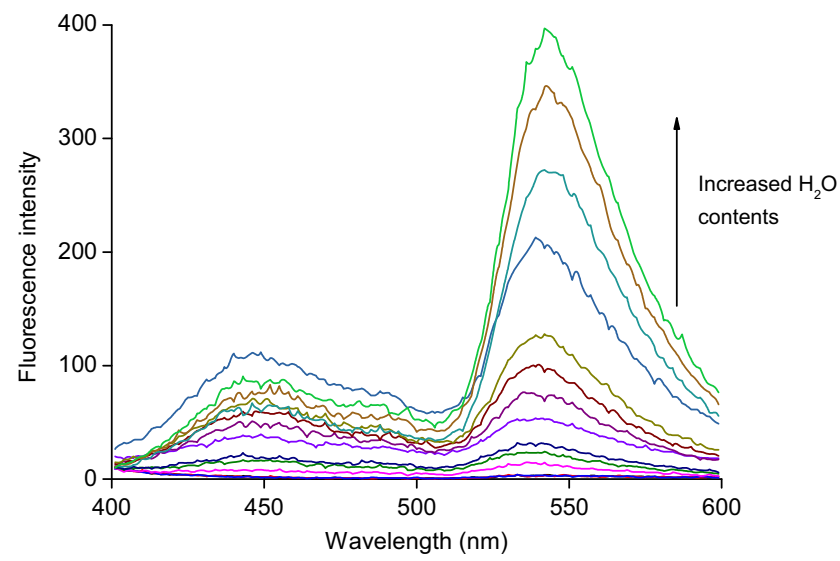

Fig. 5 a Emission spectra of 4-MU and Sudan I in hexadecane recorded for increasing contents of water. b Dependence of intensity of emission read at maximum $540 \mathrm{~nm}$ on the change of water contents in HD sample obtained ( $\mathbf{})$ in the presence of 4-MU/Sudan I, ( $)$ in the presence of 4MU/Sudan I and $10^{-5} \mathrm{M}$ octylamine, and (๑) in the presence of 4-MU/ Sudan I and $10^{-3} \mathrm{M}$ octylamine; for comparison, dependences obtained when emission intensity at $450 \mathrm{~nm}$ (i.e., using the same wavelength as in the previous work (Kłucińska et al. 2015) was plotted against water contents are shown using open symbols: ( $\square$ ) in the presence of 4-MU/ Sudan I, $(\triangle)$ in the presence of 4-MU/Sudan I and $10^{-5} \mathrm{M}$ octylamine, (O) in the presence of 4-MU/Sudan I and $10^{-3} \mathrm{M}$ octylamine. $\mathbf{c}$ Emission spectra of 4-MU and Sudan I in hexadecane recorded for increasing contents of water in the presence of $10^{-5} \mathrm{M}$ octylamine in solution 
intensity on water contents in ethanol was observed in the range from 0.05 to $6.0 \% \mathrm{~V} / \mathrm{V}$ ).

\section{Further Improvements in Sensitivity of the 4-MU/Sudan I System}

As shown above, the 4-MU/Sudan I system is highly sensitive towards the presence of water in lipophilic medium. Here, water acts as a Brønsted base converting 4-MU to its deprotonated form. However, it seems that since water is rather a weak base, it should be possible to increase the sensitivity of the system through addition of an aliphatic amine. For example, for the system containing $10^{-5} \mathrm{M}$ octylamine in hexadecane, the peak formed at $540 \mathrm{~nm}$ was, especially for higher water contents, close to $12 \% \mathrm{~V} / \mathrm{V}$, significantly higher compared to the values obtained in the absence of amine (Fig. 5b, c). For the maximum observed at $540 \mathrm{~nm}$, a linear dependence of emission intensity on water contents was obtained in the range from 0.03 to $3.33 \% V / V\left(R^{2}=0.994\right)$. In the presence of $10^{-5} \mathrm{M}$ amine in the system, the emission intensities at $450 \mathrm{~nm}$ were somewhat lower (for the same water concentrations) compared to the emission intensities obtained in the absence of amine in the sample (Fig. 5c); however, the sensitivity was higher (Fig. 5b). For the lower contents of the amine in the sample, a linear dependence of fluorescence on water contents was observed in the range from 0.03 to $3.33 \%$ $V / V\left(R^{2}=0.962\right)$. For this system, for maximum observed at $540 \mathrm{~nm}$, a linear dependence of emission on concentration was obtained even for relatively higher water contents ranging from 3.33 to $11.77 \% V / V\left(R^{2}=0.997\right)$. On the other hand, the spectrum obtained for sample containing dyes in the presence of $10^{-3} \mathrm{M}$ octylamine in hexadecane was similar to those recorded in the presence of water and in the absence of amine in the system (results not shown). In the latter case, the dependence of intensity on the water contents was linear within the concentration range from 0.03 to $3.33 V / V\left(R^{2} @ 530=0.997\right.$ and $R_{@ 450}^{2}=0.994$, respectively) (Fig. 5b).

\section{Water Contents in Vegetable Oil Samples}

The rational for the improvement of the previously proposed water content determination method based on 4-MU application was to overcome the problems that may arise when the sample is characterized with native emission at wavelengths close to $450 \mathrm{~nm}$. As model samples, vegetable (sunflower) oil and olive oil were used in this work. Sunflower oil is characterized by relatively strong fluorescence with broad emission peak from 350 to $525 \mathrm{~nm}$ (maximum around $410 \mathrm{~nm}$ ) (Fig. S4). The presence of this peak significantly hinders application of 4-MU as a water content probe. Indeed as seen in Fig. 6a, the 4-MU (only)-based system was insensitive to the increase of water contents from 0.17 to $3.25 \% \mathrm{~V} / \mathrm{V}$; further increase of water contents resulted in some increase of a

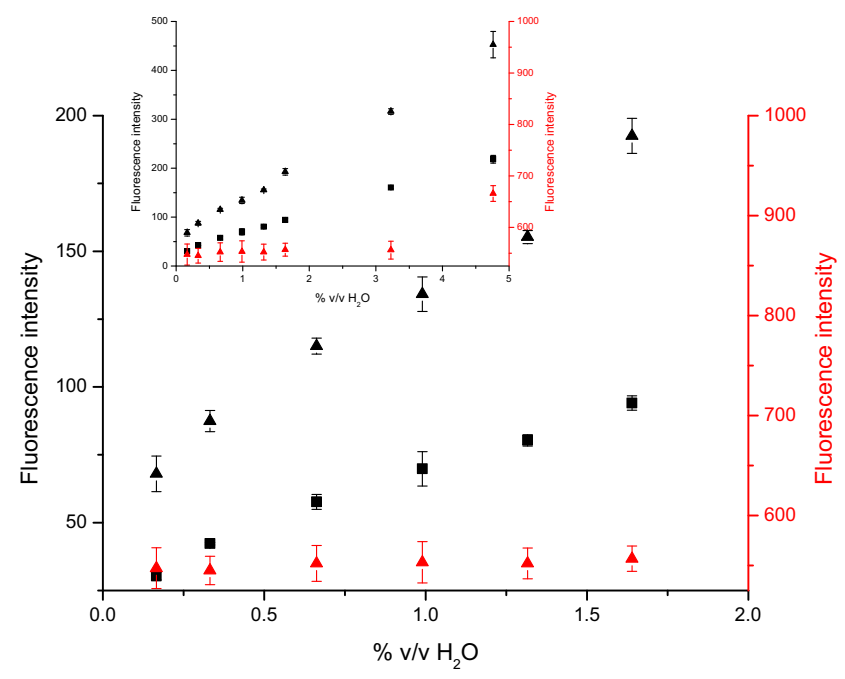

b

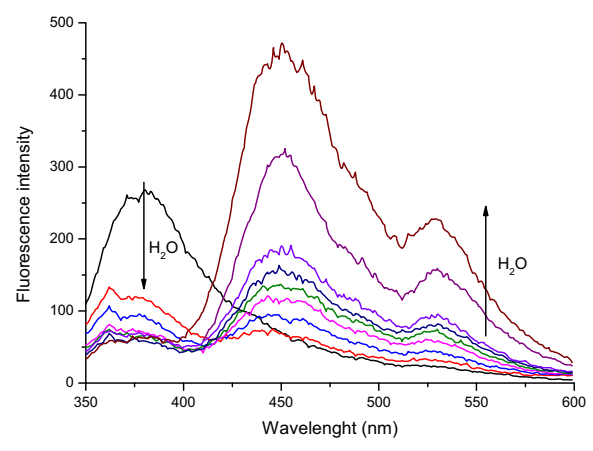

C

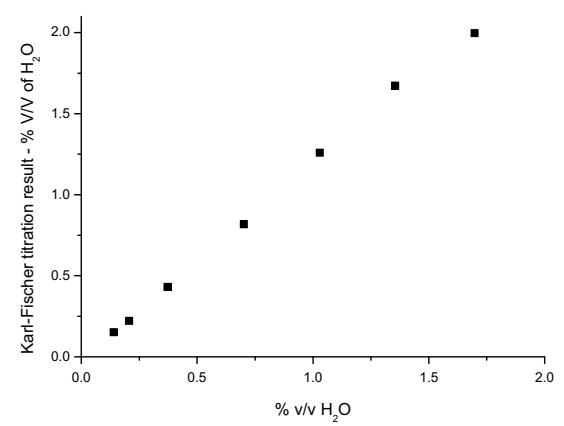

Fig. 6 a Dependence of intensity of emission on the change of water contents obtained from fluorimetric experiment in sunflower oil sample,

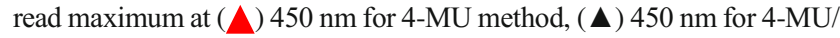
Sudan I, and (匹) $530 \mathrm{~nm}$ for 4-MU/Sudan I, inset: the same dependence for broader water contents range with $\mathrm{SD}$ (for $n=3$ nominally the same samples). b Dependence of emission spectra of 4-MU/Sudan I system in sunflower oil on water contents from 0.17 to $4.76 \% \mathrm{~V} / \mathrm{V}$ of $\mathrm{H}_{2} \mathrm{O}$. c Amount of water determined plotted against amount of water added for Karl Fischer titration of sunflower oil samples

emission intensity (Fig. 6a). On the other hand, the 4-MU/ Sudan I system, in the presence of octylamine $10^{-5} \mathrm{M}$, was characterized with significantly different dependences of emission (both read at 450 as well as $530 \mathrm{~nm}$ ) for the change 
of the water contents in the sample. For both wavelengths, the increase of emission intensity was observed even for minute concentrations of water in the sample (Fig. 6b). Addition of water to the sample resulted in decreasing of fluorescence characteristic for the sunflower oil and the formation of a new peak with two maxima: $450 \mathrm{~nm}$ (characteristic for deprotonated 4-MU form) and 530 (characteristic for 4-MU/ Sudan I system) (Fig. 6b). The maximum regarding 4-MU/ Sudan I system was observed at shorter wavelength compared to results obtained in model lipophilic solvent. For 4-MU/ Sudan I system in sunflower oil, increasing of emission intensity with increasing water contents was observed in range from 0.17 to $4.76 \%$, for both emission peaks. Linear dependence of fluorescence intensity on water contents was obtained within range from 0.17 to $4.76 \% \mathrm{~V} / \mathrm{V} \mathrm{H}_{2} \mathrm{O}$ for both maxima $\left(R^{2} @ 450=0.998\right.$ and $R^{2} @ 530=0.999$, respectively) ( $\mathrm{SD}$ of emission intensities measured for $n=3$ nominally the same samples was not exceeding $3.5 \%$ for emission read at $450 \mathrm{~nm}$ ) (Fig. 6a). In range from 0.17 to $1.64 \% V / V \mathrm{H}_{2} \mathrm{O}$, the results were comparable with those obtained using Karl Fischer titration $\left(R^{2}=0.998\right)$ (Fig. 6c). It should be stressed, however, that for the dependence of emission recorded for 4-MU/Sudan I system at $450 \mathrm{~nm}$, about two times higher sensitivity (emission change per unit concentration change) was obtained.

Olive oil used was characterized with different native emissions compared to vegetable oil; however, again, emission was observed within the range from 370 to $550 \mathrm{~nm}$, with four maxima: 380, 430, 475, or $530 \mathrm{~nm}$ Fig. S4. For this sample, application of 4-MU (only)-based approach is possible, resulting in linear dependence of emission intensity on water contents in oil olive observed within the range from 0.1 to $1.64 \%\left(R^{2} @ 450\right.$ for 4-MU $\left.=0.997\right)$ (Fig. S5). However, further increase of water contents did not result in increase of emission. On the contrary, for the 4-MU/Sudan I system, increase of water contents resulted in increased emission observed both at 450 and $530 \mathrm{~nm}$. For 4-MU/Sudan I system, linear dependence of emission intensity on water contents in the sample was observed in significantly broader range from 0.1 to $4.76 \%$ $V / V \mathrm{H}_{2} \mathrm{O}\left(R^{2} @ 450\right.$ for 4-MU/Sudan I $=0.994$ and $R^{2} @ 530$ for 4-MU/ Sudan I $=0.999)$ (Fig. S5A). Similarly as in the case of sunflower oil for the 4-MU/Sudan I system, higher sensitivity was observed for dependence observed at $450 \mathrm{~nm}$ (SD of emission intensities measured for $n=3$ nominally the same samples was not exceeding $3 \%$ for emission read at $530 \mathrm{~nm}$ ). Within the range from 0.2 to $2 \% \mathrm{~V} / \mathrm{V}$, the obtained results were comparable to those achieved using Karl Fischer titration $\left(R^{2}=0.999\right)$ (Fig. S5B).

\section{Conclusions}

The results related to fluorimetric determination of minute water content in aprotic, lipophilic, solvent are herein presented. The method is based on resonance energy transfer between the reporter dye-4-methylumbeliferone-and acceptor Sudan I. Due to applied approach, the emission signal related to water presence in the sample was observed at unique wavelength of $540 \mathrm{~nm}$, in turn on mode. The possibility of observation of emission of applied system changes related to water content at this wavelength offers significant improvement in selectivity of determination, allowing application of this method to any system that shows native emission close to $450 \mathrm{~nm}$, i.e., typical emission of applied reporter dye. The results obtained for model vegetable oils - sunflower oil and olive oil - confirmed the superiority of herein proposed method over the approach using only 4-MU in the case of samples characterized with native emission at the wavelengths close to deprotonated 4-MU emission.

Acknowledgements PR and MKC acknowledge Dr. Łukasz Dobrzycki for helpful discussions. DSc Rafał Jurczakowski's help in Karl Fischer titration is kindly acknowledged.

\section{Compliance with Ethical Standards}

Conflict of Interest Katarzyna Kłucińska declares that she has no conflict of interest. Patryk Rzepiński declares that he has no conflict of interest. Maciej Mazur declares that he has no conflict of interest. Michał K. Cyrański declares that he has no conflict of interest. Krzysztof Maksymiuk declares that he has no conflict of interest. Agata Michalska declares that she has no conflict of interest.

Ethical Approval This article does not contain any studies with human participants or animals performed by any of the authors.

Informed Consent Not applicable.

Funding Information This study was funded by the National Centre of Science (NCN, Poland), project 2014/15N/ST4/02238, in the years 2015-2017.

Open Access This article is distributed under the terms of the Creative Commons Attribution 4.0 International License (http:// creativecommons.org/licenses/by/4.0/), which permits unrestricted use, distribution, and reproduction in any medium, provided you give appropriate credit to the original author(s) and the source, provide a link to the Creative Commons license, and indicate if changes were made.

\section{References}

Bardez E, Boutin P, Valeur B (1992) Photoinduced biprotonic transfer in 4-methylumbelliferone. Chem Phys Lett 191:142-148. doi:10. 1016/0009-2614(92)85383-L

Candlin R (1956) Thermal changes in the structure of sodium sesquicarbonate. Acta Crystallographica 9:545-554. doi:10.1107/ S0365110X56001546

Dantan N, Frenzel W, Küppers S (2000) Determination of water traces in various organic solvents using Karl Fischer method under FIA conditions. Talanta 52:101-109. doi:10.1016/S0039-9140(00)00328-3

Ding L, Zhang Z, Li X, Su J (2013) Highly sensitive determination of low-level water content in organic solvents using novel 
solvatochromic dyes based on thioxanthone. Chem Commun 49: 7319-7321. doi:10.1039/C3CC43608J

Ferreira GR, Garcia HC, Couri MRC, Dos Santos HF, de Oliveiva LFC (2013) On the azo/hydrazo equilibrium in Sudan I azo dye derivatives. J Phys Chem A 117:642-649. doi:10.1021/jp310229h

Gao F, Luo F, Chen X, Yao W, Yin J, Yao Z, Wang L (2009) Fluorometric determination of water in organic solvents using europium ionbased luminescent nanospheres. Microchim Acta 166:163-167. doi:10.1007/s00604-009-0180-0

Garrigues S, Gallignani M, de la Guardia M (1993) Flow-injection determination of water in organic solvents by near-infrared spectrometry. Anal Chim Acta 281:259-264. doi:10.1016/0003-2670(93)85180$\mathrm{R}$

Gergen I, Radu F, Bordean D, Isengard H-D (2006) Determination of water content in bee's pollen samples by Karl Fischer titration. Food Control 17:176-179. doi:10.1016/j.foodcont.2004.09.018

Kłucińska K, Jurczakowski R, Maksymiuk K, Michalska A (2015) Ultrasensitive 4-methylumbelliferone fluorimetric determination of water contents in aprotic solvents. Talanta 132:392-397. doi:10. 1016/j.talanta.2014.09.018

Langhals H (1990) A simple, quick, and precise procedure for the determination of water in organic solvents. Anal Lett 23:2243-2258. doi: $10.1080 / 00032719008052562$

Li M, Paecy GE (1997) Spectrophotometric determination of trace water in organic solvents with a near infrared absorbing dye. Talanta 44: 1949-1958. doi:10.1016/S0039-9140(96)02111-X

Liu CY, Lynch V, Bard A (1997) Effect of an electric field on the growth and optoelectronic properties of quasi-one-dimensional organic single crystals of 1-(Phenylazo)-2-naphthol. J Chem Mater 9:943-949. doi:10.1021/cm9605025

Liu W, Wang Y, Jin W, Shen G, Yu R (1999) Solvatochromogenic flavone dyes for the detection of water in acetone. Anal Chim Acta 383:299307. doi:10.1016/S0003-2670(98)00789-2

Macrae CF, Bruno IJ, Chisholm JA, Edgington PR, McCabe P, Pidcock E, Rodriguez-Monge L, Taylor R, Van de Streek J, Wood PA (2008)
Mercury CSD 2.0 - new features for the visualization and investigation of crystal structures. J Appl Crystallogr 41:466-470. doi:10. 1107/S0021889807067908

Margolis SA (1995) Amperometric measurement of moisture in transformer oil using Karl Fischer reagents. Anal Chem 67:4239-4246. doi:10.1021/ac00119a006

Muthu Prabhu AA, Venkatesh G, Rajendiran N (2010) Azo-hydrazo tautomerism and inclusion complexation of 1-phenylazo-2-naphthols with various solvents and $\beta$-cyclodextrin. J Fluoresc 20:961-972. doi:10.1007/s10895-010-0642-0

Nakashima M, Sousa JA, Clapp RC (1972) Spectroscopic species of 4methylumbelliferone in water and in ethanol. Nat Phys Sci 235:1619. doi: $10.1038 /$ physci235016a0

Niu C, Li L, Qin P, Zeng G, Zhang Y (2010) Determination of water content in organic solvents by naphthalimide derivative fluorescent probe. Anal Sci 26:671-674. doi:10.2116/analsci.26.671

Oliveri AC, Wilson RB, Paul IC, Curtin DY (1989) Carbon-13 NMR and $\mathrm{X}$-ray structure determination of 1-(arylazo)-2-naphthols. Intramolecular proton transfer between nitrogen and oxygen atoms in the solid state. J Am Chem Soc 111:5525-5532. doi:10.1021/ ja00197a003

Panini P, Venugopala KN, Odhav B, Chopra D (2014) Quantitative analysis of intermolecular interactions in 7-hydroxy-4-methyl-2H-chromen-2-one and its hydrate. Proc Natl Acad Sci India Sect A 84:281295. doi:10.1007/s40010-014-0143-7

Pinheiro C, Lima JC, Parola AJ (2006) Using hydrogen bonding-specific interactions to detect water in aprotic solvents at concentrations below 50 ppm. Sensors Actuators B 114:978-983. doi:10.1016/j.snb. 2005.08.013

Sun H, Wang B, DiMagno SG (2008) A method for detecting water in organic solvents. Org Lett 10:4413-4416. doi:10.1021/o18015429

Yunxiang C, Xin J (1984) Determination of trace water in organic solvents by a spectrofluorimetric method. Talanta 31:556-558. doi: 10 . 1016/0039-9140(84)80139-3 